\title{
Randomizable Proofs and Delegatable Anonymous Credentials
}

\author{
Mira Belenkiy ${ }^{1}$, Jan Camenisch ${ }^{2}$, Melissa Chase ${ }^{3}$, Markulf Kohlweiss ${ }^{4}$, \\ Anna Lysyanskaya ${ }^{5}$, and Hovav Shacham ${ }^{6}$ \\ ${ }^{1}$ Microsoft \\ mibelenk@microsoft.com \\ ${ }^{2}$ IBM Zurich Research Laboratory \\ jca@zurich.ibm.com \\ ${ }^{3}$ Microsoft Research \\ melissac@microsoft.com \\ ${ }^{4}$ K.U. Leuven \\ mkohlwei@esat.kuleuven.be \\ ${ }^{5}$ Brown University \\ anna@cs.brown.edu \\ ${ }^{6}$ UC San Diego \\ hovav@cs.ucsd.edu
}

\begin{abstract}
We construct an efficient delegatable anonymous credentials system. Users can anonymously and unlinkably obtain credentials from any authority, delegate their credentials to other users, and prove possession of a credential $L$ levels away from a given authority. The size of the proof (and time to compute it) is $O(L k)$, where $k$ is the security parameter. The only other construction of delegatable anonymous credentials (Chase and Lysyanskaya, Crypto 2006) relies on general non-interactive proofs for NP-complete languages of size $k \Omega\left(2^{L}\right)$. We revise the entire approach to constructing anonymous credentials and identify randomizable zero-knowledge proof of knowledge systems as the key building block. We formally define the notion of randomizable non-interactive zero-knowledge proofs, and give the first instance of controlled rerandomization of non-interactive zero-knowledge proofs by a third-party. Our construction uses Groth-Sahai proofs (Eurocrypt 2008).
\end{abstract}

\section{Introduction}

Access control is one of the most fundamental problems in security. We frequently need to answer the question: does the person requesting access to a resource possess the required credentials? A credential typically consists of a certification chain rooted at some authority responsible for managing access to the resource and ending at the public key of the user in question. The user presents the credential and demonstrates that he knows the corresponding secret key. Sometimes, the trusted authority issues certificates directly to each user (so the length of each certification chain is 1). More often, the authority delegates responsibility. A system administrator allows several webmasters to use

S. Halevi (Ed.): CRYPTO 2009, LNCS 5677, pp. 108 125, 2009

(C) International Association for Cryptologic Research 2009 
his server. A webmaster can create several forums, with different moderators for each forum. Moderators approve some messages, reject others, and even give favored users unlimited posting privileges. Imagine the burden on the system administrator if he had to approve every single moderator and user for every single forum.

We want cryptographic credentials to follow the same delegation model as access control follows in the real world. The system administrator can use his public key to sign a webmaster's public key, creating a credential of length 1 . In general, a user with a level $L$ credential can sign another user's public key and give him his credential chain, to create a level $L+1$ credential.

The design of an anonymous delegatable credential scheme in which participants can obtain, delegate, and demonstrate possession of credential chains without revealing any additional information about themselves is a natural and desirable goal. Our main contribution is the first efficient delegatable anonymous credential scheme. The only known construction of delegatable anonymous credentials, due to Chase and Lysyanskaya CL06, needs $k^{\Omega(L)}$ space to store a certification chain of length $L$ (for security parameter $k$ ), and therefore could not tolerate non-constant $L$. Our solution is practical: all operations on chains of length $L$ need $\Theta(k L)$ time and space.

Pseudonymous systems. Prior work on anonymous credentials CL02b, BCKL08 created systems where each user has one secret key but multiple "public keys." Given a secret key $s k_{A}$, Alice can create a new public key by choosing a random value open and publishing a commitment $p k_{A}=\operatorname{Commit}\left(s k_{A}\right.$, open $)$. Alice could register $p k_{A}$ with Oliver and $p k_{A}^{\prime}$ with Olga.

Oliver can give Alice a credential by signing the statement that the value in commitment $p k_{A}$ has some attribute. Alice can then show $p k_{A}^{\prime}$ to Olga and prove that Oliver signed that the value in $p k_{A}^{\prime}$ had that attribute. This works because $p k_{A}$ and $p k_{A}^{\prime}$ are commitments to the same value $s k_{A}$. The chief building block of an anonymous credential scheme is a signature scheme that lends itself to the design of efficient protocols for (1) obtaining a signature on a committed value; and (2) proving that a committed value has been signed.

Why delegation is a challenging problem. There is no straightforward transformation of anonymous credential schemes Cha85, Bra99, LRSW99, CL01, BCKL08 into delegatable schemes. Suppose instead of giving Alice a direct credential, Oliver delegates his own credential to Alice. If Oliver gives Alice a sig on Oliver's secret key, then Alice could learn who gave Oliver his credential. Generalizing this approach would reveal to Alice the identity of every person in Oliver's credential chain.

Our approach. Instead of giving Alice his signature, Oliver gives Alice a noninteractive proof-of-knowledge of the signature. We show how Alice can (1) delegate the credential by extending the proof and (2) rerandomize the proof every time she shows (or extends it) to preserve her anonymity.

Let's say Oliver is a credential authority and Alice wants to obtain the credential directly from Oliver (so her certification chain will be of length 1 ). Under 
the old approach, they would run a secure two-party protocol as a result of which Alice obtains a signature $\sigma_{p k_{O}}\left(s k_{A}\right)$ on $s k_{A}$, while Oliver gets no output. Under the new approach, Alice's output is $\left(C_{A}, \pi_{A}\right)$, where $C_{A}$ is a commitment to her secret key $s k_{A}$, and $\pi_{A}$ is a proof of knowledge of Oliver authenticating the contents of $C_{A}$. Note that a symmetric authentication scheme is sufficient because no one ever sees the authenticator; all verification is done on the proof of knowledge. The symmetric key $s k_{O}$ is still known only to Oliver; we create a "public" key $C_{O}$ that is simply a commitment to $s k_{O}$.

How can Alice use this credential anonymously? If the underlying proof system is malleable in just the right way, then given $\left(C_{A}, \pi_{A}\right)$ and the opening to $C_{A}$, Alice can compute $\left(C_{A}^{\prime}, \pi_{A}^{\prime}\right)$ such that $C_{A}^{\prime}$ is another commitment to her $s k_{A}$ that she can successfully open, while $\pi_{A}^{\prime}$ is a proof of knowledge of Oliver authenticating the contents of $C_{A}^{\prime}$. Malleability is usually considered a bug rather than a feature. However, in combination with the correct extraction properties, we still manage to guarantee that these randomizable proofs give us a useful building block for the construction.

How does Alice delegate her credential to Bob? Alice and Bob can run a secure protocol as a result of which Bob obtains $\left(C_{B}, \pi_{B}\right)$ where $C_{B}$ is a commitment to Bob's secret key $s k_{B}$ and $\pi_{B}$ is a proof of knowledge of an authenticator issued by the owner of $C_{A}^{\prime}$ on the contents of $C_{B}$. Now, essentially, the set of values $\left(C_{A}^{\prime}, C_{B}, \pi_{A}^{\prime}, \pi_{B}\right)$ together indicate that the owner of $C_{A}^{\prime}$ got a credential from Oliver and delegated to the owner of $C_{B}$, and so it constitutes a proof of possession of a certification chain. Moreover, it hides the identity of the delegator Alice! Now Bob can, in turn, use the randomization properties of the underlying proof system to randomize this set of values so that it becomes unlinkable to his original pseudonym $C_{B}$; he can also, in turn, delegate to Carol.

Randomizable proof systems. The key to our construction is a randomizable proof system that lets the prover (1) randomize the proof without knowing the witness, and (2) control the outcome of the randomization process. This is a fundamentally new notion, and one we think will be of independent interest. We give a formal definition and show that we can instantiate it by adding a randomization procedure to the pairing-based proof system of Groth and Sahai GS08. Our use of pairings is not merely a matter of efficiency - we do not know of any proof system based on general assumptions that can be randomized.

In fact the Groth-Sahai proofs allow us to go beyond merely randomizing the proof to actually change the statements we are proving. What do we mean by this? Groth-Sahai proofs make statements about the values inside commitments. Let $C=\operatorname{Commit}(x$, open $)$. A prover who knows $(x$, open $)$ can choose a new value open' so that in the rerandomized proof, $C$ is transformed to $C^{\prime}=\operatorname{Commit}\left(x\right.$, open $\left.^{\prime}\right)$. Otherwise, the prover can choose whether to leave $C$ unchanged or randomize it to $C^{\prime}$ that uses a some random open' unknown to the prover. This fine level of control together with the basic randomization property gives a very useful building block, which is crucial in our application.

There has been prior work on some related notions: Burmester et al $\mathrm{BDI}^{+} 99$ show a third party can help randomize proofs during the execution of an 
interactive protocol to prevent subliminal channels. De Santis and Yung [DSY90] propose the notion of meta proofs, in which anyone who holds a proof for a given statement can generate a proof that there exists a proof for the statement. Neither of these approaches work for our scenario because we need to randomize non-interactive proofs, and, unlike a meta-proof, the randomized proof must be indistinguishable from the original.

Our delegatable credentials construction. We construct delegatable credentials using randomizable proofs. By concatenating rerandomized credential chains, we can create a credential chain of length $L$ that takes $O(L)$ space. Our strong anonymity properties are an immediate consequence of rerandomization: each showing of the credential is unlinkable and users do not learn the identities of delegators in their own credential chain.

Our solution (1) prevents adversarial users from mixing and matching pieces of different credential chains to create unauthorized credential chains and (2) protects the user's anonymity even when the adversary delegates to the user. We solve the second problem by creating an authentication scheme (symmetric signature scheme) that is secure even when the adversary gets a signature on the user's secret key.

Attributes. Our delegatable anonymous credentials system lets users add humanreadable attributes to each credential. Oliver can give Alice a level 1 credential with attribute "webmaster of Crypto Forum". Alice can then delegate her credential to Bob with attribute "moderator of Crypto Forum". As a result, Bob can log on to the server anonymously and prove that the "webmaster of Crypto Forum" made him the "moderator of Crypto Forum". Our construction lets users add as many attributes as they want to each credential, allowing for the expressibility that we see in modern (non-anonymous) access control systems.

Advanced abuse prevention mechanisms. Our construction shows how to efficiently implement maximum anonymity at all levels and all roles in the delegation chain - with the exception of the credential authority. Some applications will not require this full anonymity. Indeed, a large number of abuse prevention mechanisms for anonymous credentials (anonymity revocation CL01, credential revocation [CL02a, limited show $\left[\mathrm{CHK}^{+} 06\right]$ ) aim at striking a balance between privacy and accountability. Concerning our own scheme we make three simple observations: (i) global traceability can be achieved by providing a trusted tracing authority with the extraction trapdoors for the common parameters of the Groth Sahai (GS) proof system; (ii) at the last level of the delegation chain, we can make use of all abuse prevention mechanisms known for traditional anonymous credentials; (iii) many abuse prevention mechanisms known from the literature can be adapted to our construction by replacing traditional sigma proofs Dam02 with GS proofs GS08.

Other related work. At first glance, our delegatable credentials scenario might resemble the HIBE or HIBS settings GS02, BBG05, where a root delegator can issue decryption or signing keys to recipients, who in turn can delegate subkeys to lower level participants. There are two key differences between such a 
HIBE/HIBS scheme and anonymous credential schemes: (1) In HIBE/HIBS two users with the same attributes are completely interchangeable while an anonymous credentials system gives them distinct sets of pseudonyms and (2) anonymous credentials allow a user to show that he has obtained valid credentials from two independent authorities.

In a somewhat different direction, Barak Bar01 presented a general (inefficient) construction for delegatable signatures. In that work, the goal was a signature scheme which would allow the signer to delegate signing rights for a restricted message space, such that signatures generated with the delegated key are indistinguishable from the originals. In our setting, we want the opposite: once we delegate a credential, the delegatee should be able to issue lower level credentials to any users he chooses, however we require that credentials at different levels be clearly distinguishable. Finally, the definition in Bar01 does not consider anonymity between the delegator and the delegatee, while we do.

Our contribution and organization of the paper. We (1) define and construct a randomizable NIZKPK (Section 2) and (2) define and construct an efficient delegatable anonymous credential system (Section 3). We also create an appropriate message authentication scheme and some other additional building blocks for the delegatable credentials scheme. We show how these building blocks can be instantiated under appropriate assumptions about groups with bilinear maps.

\section{Randomizable NIZK Proof Systems}

Let $R($ params $, y, w)$ be any polynomial-time computable relation. A noninteractive proof system for relation $R$ allows the prover to convince a verifier that for some instance $y$ there exists a witness $w$ such that $R($ params, $y, w)$, where params is a common (public) reference string. The prover generates a proof $\pi \leftarrow \operatorname{Prove}($ params, $y, w)$, the verifier checks it via VerifyProof (params, $y, \pi)$. A trusted third party runs params $\leftarrow \operatorname{Setup}\left(1^{k}\right)$ once to initialize the system.

Informally, zero-knowledge captures the notion that a verifier learns nothing from the proof but the truth of the statement. Witness indistinguishability merely guarantees that the verifier learns nothing about which witness was used in the proof. Soundness means an adversary cannot convince an honest verifier of a false statement. Completeness means all honest verifiers accept all correctly computed proofs. See GMR89, Gol00, BFM88, FLS99 for formal definitions.

We define randomizable proof systems, which have an additional algorithm RandProof that takes as input a proof $\pi$ for instance $y$ in relation $R$, and produces a new proof for the same statement $y$. The resulting proof must be indistinguishable from a new proof for $y$. We allow the adversary to choose the instance $y$, the proof $\pi$ that is used as input for RandProof, and the witness $w$ that is used to form a new proof of the same statement. Formally:

Definition 1. We say that Setup, Prove, VerifyProof, RandProof constitute a randomizable proof system if the following property holds. For all ppt. $\left(\mathcal{A}_{1}, \mathcal{A}_{2}\right)$ there exists a negligible function $\nu$ such that: 


$$
\begin{aligned}
& \operatorname{Pr}\left[\text { params } \leftarrow \operatorname{Setup}\left(1^{k}\right) ;(y, w, \pi, \text { state }) \leftarrow \mathcal{A}_{1}(\text { params }) ;\right. \\
& \pi_{0} \leftarrow \operatorname{Prove}(\text { params }, y, w) ; \pi_{1} \leftarrow \text { RandProof }(\text { params }, y, \pi) ; \\
& b \leftarrow\{0,1\} ; b^{\prime} \leftarrow \mathcal{A}_{2}\left(\text { state }, \pi_{b}\right): \\
& \left.R_{L}(y, w) \wedge \operatorname{VerifyProof}(\text { params }, y, \pi)=1 \wedge b=b^{\prime}\right] \leq 1 / 2+\nu(k) .
\end{aligned}
$$

\subsection{Instantiating a Randomizable Proof System}

Randomization is a fundamentally new property. It is not clear how one might randomize proofs in any of the existing NIZK proof systems BDMP91, KP98, FLS99 without knowing the witness. The one exception is the recent proof system of Groth and Sahai GS08 (an extension of GOS06]), which gives witness indistinghishable (and in some cases zero-knowledge) NIPKs. We will show how to add a randomization procedure to Groth-Sahai proofs.

Summary of Groth-Sahai proofs. Let params ${ }_{B M}=\left(p, G_{1}, G_{2}, G_{T}, e, g, h\right)$ be the setup for pairing groups of prime order $p$, with pairing $e: G_{1} \times G_{2} \rightarrow G_{T}$, and $g, h$ generators of $G_{1}, G_{2}$ respectively 1

The instance consists of the coefficients of a pairing product equation:

$\left\{a_{q}\right\}_{q=1 \ldots Q} \in G_{1},\left\{b_{q}\right\}_{q=1 \ldots Q} \in G_{2}, t \in G_{T}$, and $\left\{\alpha_{q, m}\right\}_{q=1 \ldots Q, m=1 \ldots M}$, $\left\{\beta_{q, n}\right\}_{q=1 \ldots Q, n=1 \ldots N} \in Z_{p}$. The prover knows $\left\{x_{m}\right\}_{m=1}^{M},\left\{y_{n}\right\}_{n=1}^{N}$ that satisfy the pairing product equation $\prod_{q=1}^{Q} e\left(a_{q} \prod_{m=1}^{M} x_{m}^{\alpha_{q, m}}, b_{q} \prod_{n=1}^{N} y_{n}^{\beta_{q, n}}\right)=t$.

The prover creates perfectly binding, computationally hiding commitments $\left\{c_{m}\right\}_{m=1 \ldots M}$ and $\left\{d_{n}\right\}_{n=1 \ldots N}$ for all values $x_{m}, y_{n}$ in $G_{1}$ and $G_{2}$ respectively. The instance is the pairing product equation (e.g. its coefficients) and the above commitments, while the witness, known only to the prover, is the values and openings of these commitments.

We now describe how to construct the proof. Let $M_{1}, M_{2}$, and $M_{T}$ be $R$ modules for some $\operatorname{ring} R$, and let $E: M_{1} \times M_{2} \rightarrow M_{T}$ be a bilinear map. Also let $\mu_{1}, \mu_{2}, \mu_{T}$ be efficiently computable embeddings that map elements of $G_{1}, G_{2}, G_{T}$ into $M_{1}, M_{2}, M_{T}$, respectively. The public parameters params $_{P K}$ contain elements $u_{1}, \ldots, u_{I} \in M_{1}, v_{1}, \ldots, v_{J} \in M_{2}$ and values $\eta_{h, i, j}, 1 \leq i \leq I, 1 \leq j \leq J$, and $1 \leq h \leq H$.

To create a Groth-Sahai commitment to $x \in G_{1}$, choose random opening open $=\left(r_{1}, \ldots, r_{I}\right) \leftarrow R^{I}$, and compute $c=\mu_{1}(x) \cdot \prod_{i=1}^{I} u_{i}^{r_{i}}$. Elements $y \in G_{2}$ are committed to in the same way using $\mu_{2}$ and $v_{1}, \ldots, v_{J} \in M_{2}$, and an opening vector open $\in R^{J}$. For simplicity we assume that GSCommit $\left(\right.$ params $_{P K}, m$, open $)$ first determines whether $m \in G_{1}$ or $m \in G_{2}$ and then follows the appropriate instructions.

Groth and Sahai GS08] show how to efficiently compute proofs $\left\{\pi_{i}\right\}_{i=1}^{I}$, $\left\{\psi_{j}\right\}_{j=1}^{J}$ that prove that the openings of the $c_{m}$ and $d_{n}$ satisfy a pairing product equation. The verifier computes, for all $1 \leq q \leq Q, \hat{c}_{q} \leftarrow \mu_{1}\left(a_{q}\right) \cdot \prod_{m=1}^{M} c_{m}^{\alpha_{q, m}}$

\footnotetext{
${ }^{1}$ For simplicity, we do not consider Groth-Sahai proofs for composite order groups.
} 
and $\hat{d}_{q} \leftarrow \mu_{2}\left(b_{q}\right) \cdot \prod_{n=1}^{N} d_{n}^{\beta_{q, n}}$. Then the verifier checks that $\prod_{q=1}^{Q} E\left(\hat{c}_{q}, \hat{d}_{q}\right)=$ $\mu_{T}(t) \cdot \prod_{i=1}^{I} E\left(u_{i}, \pi_{i}\right) \cdot \prod_{j=1}^{J} E\left(\psi_{j}, v_{j}\right)$.

Randomizing Groth-Sahai proofs. RandProof gets as input an instance with the $a_{q}, b_{q}, t, \alpha_{q, m}, \beta_{q, n}$ values as well as the proof $\left[\left(\pi_{1}, \ldots, \pi_{I}, \psi_{1}, \ldots, \psi_{J}\right), \Pi\right] . \Pi$ contains the internal commitments $c_{1}, \ldots, c_{M}$ and $d_{1}, \ldots, d_{N}$.

The algorithm first chooses randomization exponents $\left(s_{1,1}, \ldots, s_{M, I}\right)$ and $\left(z_{1,1}, \ldots, z_{N, J}\right)$ at random from $Z_{p}$. It then rerandomizes the commitments $c_{m}$ and $d_{n}$ to $c_{m}^{\prime}=c_{m} \cdot \prod_{i=1}^{I} u_{i}^{s_{m, i}}$ and $d_{n}^{\prime}=d_{n} \cdot \prod_{j=1}^{J} v_{j}^{z_{n, j}}$. Then it computes $\hat{s}_{q, i}=\sum_{m=1}^{M} s_{m, i} \cdot \alpha_{q, m}, \hat{z}_{q, j}=\sum_{n=1}^{N} z_{n, j} \cdot \beta_{q, n}$, and $D_{q}^{\prime} \leftarrow \mu_{2}\left(b_{q}\right) \cdot \prod_{n=1}^{N} d_{n}^{\prime \beta_{q, n}}$ and $C_{q} \leftarrow \mu_{1}\left(a_{q}\right) \cdot \prod_{m=1}^{M} c_{m}^{\alpha_{q, m}}$. Next, the prover sets $\pi_{i}^{\prime} \leftarrow \pi_{i} \cdot \prod_{q=1}^{Q}\left(D_{q}^{\prime}\right)^{\hat{s}_{q, i}}$ and $\psi_{j}^{\prime} \leftarrow \psi_{j} \cdot \prod_{q=1}^{Q}\left(C_{q}\right)^{\hat{z}_{q, j}}$. These $\pi_{i}^{\prime}$ and $\psi_{j}^{\prime}$ will satisfy the verification equation for the new commitments.

Now the prover must make a certain technical step to fully randomize the proof. Intuitively, for every set of commitments, there are many proofs $\left(\pi_{1}, \ldots, \pi_{I}\right.$,

$\left.\psi_{1}, \ldots, \psi_{J}\right)$ that can satisfy the verification equation. Given one such proof, we can randomly choose another: The prover chooses $t_{i, j}, t_{h} \leftarrow R$, and multiplies each $\pi_{i}^{\prime}:=\pi_{i}^{\prime} \cdot \prod_{j=1}^{J} v_{j}^{t_{i, j}}$ and each $\psi_{j}^{\prime}:=\psi_{j}^{\prime} \cdot \prod_{i=1}^{I} u_{i}^{\sum_{h=1}^{H} t_{h} \eta_{h, i, j}} \prod_{i=1}^{I} u_{i}^{t_{i, j}}$. See [GS08] for a detailed explanation.

The algorithm outputs the new proof $\left[\left(\pi_{1}^{\prime}, \ldots, \pi_{I}^{\prime}, \psi_{1}^{\prime}, \ldots, \psi_{J}^{\prime}\right), \Pi^{\prime}\right]$ where $\Pi^{\prime}$ contains the internal commitments $c_{1}^{\prime}, \ldots, c_{M}^{\prime}$ and $d_{1}^{\prime}, \ldots, d_{N}^{\prime}$. See full version $\mathrm{BCC}^{+} 08$ for details. A similar approach works for composite order groups.

Composable Proofs. Groth-Sahai proofs are composable witness indistinguishable, and in some cases composable zero-knowledge. To simplify our definitions and proofs, we use a similar notion for randomizability.

In a composable (under the definition of Groth and Sahai GS08) noninteractive proof system there exists an algorithm SimSetup that outputs params together with a trapdoor sim, such that params output by SimSetup is indistinguishable from those output by Setup. Composable witness-indistinguishability (or zero-knowledge) requires that, under these parameters, the witness-indistinguishability (resp. zero-knowledge) property holds even when the adversary is given the trapdoor sim. Groth-Sahai commitments are perfectly hiding under the simulated parameters. (Under the honest parameters they are perfectly binding.) In the same spirit, we say the composable randomizability property must hold even when the distinguisher is given the trapdoor sim.

\subsection{Malleable Proofs and Randomizable Commitments}

For our application, randomizing proofs is not sufficient. We also need to randomize (anonymize) the statement that we are proving. Consider a family of transformations $\left\{Y_{s}, P_{s}\right\}_{s \in S}$ that transform the instance and the proof respectively (for us, $S$ is the set of all possible commitment openings). We require that $\forall(y, \pi), \forall s \in S$, if $\pi$ is a valid proof for $y$, then $P_{s}(\pi)$ is a valid proof for $Y_{s}(y)$. 
Definition 2. We say that Setup, Prove, VerifyProof, RandProof, $\left\{Y_{s}, P_{s}\right\}_{s \in S}$, constitute a $Y$-malleable randomizable proof system, if for all ppt. $\mathcal{A}$ there exists a negligible $\nu$ such that:

$$
\begin{aligned}
& \operatorname{Pr}\left[\text { params } \leftarrow \operatorname{Setup}\left(1^{k}\right) ;(y, \pi, s) \leftarrow \mathcal{A}(\text { params }):\right. \\
& \left.\quad \text { VerifyProof }(\text { params }, y, \pi)=1 \wedge \operatorname{VerifyProof}\left(\text { params }, Y_{s}(y), P_{s}(\pi)\right)=0\right]=\nu(k) .
\end{aligned}
$$

If we apply RandProof to $P_{s}(\pi)$, then the result will be indistinguishable from a random fresh proof for $Y_{s}(y)$.

Groth-Sahai proofs can be used to prove that the values in a given set of commitments form a solution to a specific set of pairing product equations; the commitments can be part of the proof or the instance $y$. In our application, we will need to anonymize not only the proof, but also the commitments in the instance.

Suppose a prover wants to show that some Condition holds for the values inside commitments $C_{1}, \ldots, C_{n}$. Then the instance is $y=$ (Condition, $C_{1}, \ldots$, $\left.C_{n}\right)$, and the witness is $w=\left(x_{1}\right.$, open $_{1}, \ldots, x_{n}$, open $\left.{ }_{n}, z\right)$, where $\left(x_{i}\right.$, open $\left._{i}\right)$ is the opening of commitment $C_{i}$, while $z$ is some value that has nothing to do with the commitments. We define the relation $R=\left\{(\right.$ params, $y, w) \mid C_{1}=$ Commit $\left({\left.\text { params }, x_{1}, \text { open }_{1}\right) \wedge \ldots \wedge C_{n}=\operatorname{Commit}\left(\text { params }_{1} x_{n} \text {, open }\right.}_{n}\right) \wedge$ Condition (params, $\left.\left.x_{1}, \ldots, x_{n}, z\right)\right\}$. A proof system supports randomizable commitments if there exist efficient algorithms $Y$ and $P$, such that on input $(s, y, \pi)$, where $s=$ (open $_{1}^{\prime}, \ldots$, open $_{n}^{\prime}$ ) and $\pi \leftarrow$ Prove $($ params, $y, w)$, (1) $Y(s, y)$ outputs instance $y^{\prime}=\left(\right.$ Condition, $\left.C_{1}^{\prime}, \ldots, C_{n}^{\prime}\right)$, where $C_{i}^{\prime}=\operatorname{Commit}\left(\right.$ params,$x_{i}$, open open $\left._{i}^{\prime}\right)$, (2) $P(s, \pi)$ outputs a proof $\pi^{\prime}$ for instance $y^{\prime}$, and (3) $Y$ and $P$ fulfill the malleability requirements of Definition 2 .

Lemma 1. The Groth-Sahai proof system is malleable with respect to the randomness in the commitments. See full version $\mathrm{BCC}^{+} 08$ for details.

Remark 1. To simplify notation, RandProof will take $s=\left(\right.$ open $_{1}^{\prime}, \ldots$, open $\left.n_{n}^{\prime}\right)$ as input, apply $P_{s}$, and then run the randomization algorithm. To leave $C_{i}$ unchanged, we set open ${ }_{i}^{\prime}=0$.

\subsection{Partially Extractable Non-interactive Proofs of Knowledge}

A NIPK system is a non-interactive proof system that is extractable. We recall the notion of f-extractability [BCKL08, which is an extension of the original definition of extractability SCP00]. In an extractable proof system, there exists a ppt. extractor (PKExtractSetup, PKExtract). PKExtractSetup $\left(1^{k}\right)$ outputs $(t d$, params $)$ where params is distributed identically to the output of Setup $\left(1^{k}\right)$. For all polynomial time adversaries $\mathcal{A}$, the probability that $\mathcal{A}\left(1^{k}\right.$, params $)$ outputs $(y, \pi)$ such that VerifyProof $($ params $, y, \pi)=\operatorname{accept}$ and PKExtract $(t d, y, \pi)$ fails to extract a witness $w$ such that $R($ params, $y, w)=$ accept is negligible in $k$. We have perfect extractability if this probability is $0 . f$-Extractability means that the extractor PKExtract only has to output a $w^{\prime}$ such that $\exists w$ : 
$R($ params $, y, w)=$ accept $\wedge w^{\prime}=f($ params,$w)$. If $f($ params,$\cdot)$ is the identity function, we get the usual notion of extractability.

Let $C$ be an unconditionally binding commitment. By ' $x$ in $C$ ' we mean $\exists$ open : $C=$ Commit (params, $x$, open). We use NIPK notation CS97, BCKL08, to denote an $f$-extractable NIPK for instance $\left(C_{1}, \ldots, C_{n}\right.$, Condition) with witness $\left(x_{1}\right.$, open $_{1}, \ldots, x_{n}$, open $\left._{n}, z\right)$ :

$$
\pi \leftarrow \operatorname{NIPK}\left[x_{1} \text { in } C_{1}, \ldots, x_{n} \text { in } C_{n}\right]\left\{\left(f\left(\text { params },\left(x_{1}, \text { open }_{1}, \ldots, x_{n}, \text { open }_{n}, z\right)\right)\right):\right.
$$

Condition $\left(\right.$ params $\left.\left., x_{1}, \ldots, x_{n}, z\right)\right\}$.

The $f$-extractability property ensures that if VerifyProof accepts then we can extract $f\left(\right.$ params $\left(x_{1}\right.$, open $_{1}, \ldots, x_{n}$, open $\left.\left._{n}, z\right)\right)$ from $\pi$, such that $x_{i}$ is the content of the commitment $C_{i}$, and Condition (params, $\left.x_{1}, \ldots, x_{n}, z\right)$ is satisfied.

In our notation, $\pi \in$ NIPK $[\ldots\}$ means that VerifyProof accepts the proof $\pi$ for instance $\left(C_{1}, \ldots, C_{n}\right.$, Condition). To further abbreviate notation, we omit params and assume that Condition is clear from the context, and so the sole inputs to VerifyProof are $\left(C_{1}, \ldots, C_{n}\right)$ and $\pi$. If the proof is zero-knowledge instead of merely witness indistinguishable, we will write NIZKPK.

The concatenation of two proofs $\pi$ and $\pi^{\prime}$ is a proof $\pi \circ \pi^{\prime}$ that combines all the commitments and proves the AND of the two conditions. If a proof $\pi$ proves a condition about a set of commitments $\mathcal{C}$, a projection $\pi^{\prime}=\pi \circ \mathcal{S}$ proves a condition about the contents of the subset $\mathcal{C} \backslash \mathcal{S}$ of commitments. A projected proof $\pi^{\prime}$ is obtained by removing the commitments in $\mathcal{S}$ from the instance and appending them to the proof.

Groth-Sahai proofs give us NIPK of the form:

$$
\begin{aligned}
\text { NIPK }_{\mathrm{GS}}\left[\left\{x_{m} \text { in } c_{m}\right\}_{m=1}^{M},\left\{y_{n} \text { in } d_{n}\right\}_{n=1}^{N}\right]\left\{\left(x_{1}, \ldots, x_{M}, y_{1}, \ldots, y_{N}\right):\right. \\
\\
\left.\prod_{q=1}^{Q} e\left(a_{q} \prod_{m=1}^{M} x_{m}^{\alpha_{q, m}}, b_{q} \prod_{n=1}^{N} y_{n}^{\beta_{q, n}}\right)=t\right\} .
\end{aligned}
$$

\section{Delegatable Anonymous Credentials}

An anonymous delegatable credential system has only one type of participant: users. Each user has a single secret key and uses it to generate different pseudonyms. User $A$ with secret key $s k_{A}$ can be known to user $O$ as $N y m_{A}^{(O)}$ and to user $B$ as $N y m_{A}^{(B)}$. Any user $O$ can become an originator of a credential; all he needs to do is publish one of his pseudonyms $N y m_{O}$ as his public key. If authority $O$ issues user $A$ a credential for $N y m_{A}^{(O)}$, then user $A$ can prove to user $B$ that $N y m_{A}^{(B)}$ has a credential from authority $O$. Credentials received directly from the authority are level 1 credentials, credentials that have been delegated once are level 2 credentials, etc. A delegatable credential system consists of the following algorithms: 
Setup $\left(1^{k}\right)$ outputs the trusted public parameters of the system, params $_{D C}$.

Keygen $\left(\right.$ params $\left._{D C}\right)$ creates the secret key of a party in the system.

Nymgen $\left(\right.$ params $\left._{D C}, s k\right)$. On each run, the algorithm outputs a new pseudonym Nym with auxiliary info aux (Nym) for secret key $s k 2$

Issue $\left(\right.$ params $_{D C}$, Nym $_{O}$, sk $_{I}, N_{y m_{I}}$, aux $\left(\right.$ Nym $\left._{I}\right)$, cred $\left., N y m_{U}, L\right)$

$\leftrightarrow$ Obtain $\left(\right.$ params $\left._{D C}, \operatorname{Nym}_{O}, \operatorname{sk}_{U}, N y m_{U}, \operatorname{aux}\left(N y m_{U}\right), N y m_{I}, L\right)$ are the interactive algorithms that let a user $I$ issue a level $L+1$ credential to a user $U$. The pseudonym $N y m_{O}$ is the authority's public key, $s k_{I}$ is the issuer's secret key, Nym $_{I}$ is the issuer's pseudonym with auxiliary information aux $\left(N y m_{I}\right)$, cred is the issuer's level $L$ credential rooted at $N y m_{O}, s k_{U}$ is the user's secret key, and $N y m_{U}$ is the user's pseudonym with auxiliary information $\operatorname{aux}\left(\operatorname{Nym}_{U}\right)$. If $L=0$ then cred $=\epsilon$. The issuer gets no output, and the user gets a credential cred $_{U}$.

CredProve $\left(\right.$ params $_{D C}$, Nym $_{O}$, cred, sk, Nym, aux $\left.(N y m), L\right)$. Takes as input a level $L$ credential cred from authority $N y m_{O}$, outputs a value credproof.

CredVerify $\left(\right.$ params $_{D C}$, Nym $_{O}$, credproof $\left., N y m, L\right)$. Outputs accept if credproof is a valid proof that the owner of pseudonym $N y m$ possesses a level $L$ credential with root $\mathrm{Nym}_{O}$ and reject otherwise.

\subsection{Security Definition of Delegatable Credentials}

We formally define a secure delegatable credential system in the full version $\mathrm{BCC}^{+} 08$. Intuitively, the algorithms Setup, Keygen, Nymgen, VerifyAux, Issue, Obtain, CredProve, and CredVerify constitute a secure anonymous delegatable credential scheme if the following properties hold:

Correctness. We say that a credential cred is a proper credential, if for all of the user's pseudonyms, CredProve always creates a proof that CredVerify accepts. The delegatable credential system is correct if an honest user and an honest issuer can run Obtain $\leftrightarrow$ Issue and the honest user gets a proper credential.

Anonymity. The adversary's interactions with the honest parties in the real game should be indistinguishable from some ideal game in which pseudonyms, credentials and proofs are independent of the user's identity and delegation chain. The adversary should not even recognize a credential he delegated.

There must exist a simulator (SimSetup, SimProve, SimObtain, Simlssue). SimSetup produces parameters indistinguishable from those output by Setup, along with some simulation trapdoor sim. Under these parameters, we require that the following properties hold when even the adversary is given sim:

- Nym is distributed independently of $s k$.

- No adversary can tell if it is interacting with Issue run by an honest party with a proper credential, or with Simlssue which is not given the credential and the issuer's secret key, but only the name of the authority, the length of the credential chain, and the pseudonyms of the issuer and user.

${ }^{2}$ We do not address how to prove ownership of a pseudonym; in our constructions this involves interactively proving knowledge of the opening of a commitment. 
- No adversary can tell if it is interacting with Obtain run by an honest party with secret $s k$, or with SimObtain that is only given the authority, the length of the credential chain, and the pseudonyms of the issuer and user.

- The simulator SimProve can output a fake credproof that cannot be distinguished from a real credential, even when SimProve is only told the authority, the length of the credential chain, and the pseudonym of the user.

Remark 2. Our definition implies the more complex but weaker definition in which the adversary only controls the public inputs to the algorithm. Our definition is easier to work with as we need only consider one protocol at a time, and only a single execution of each protocol.

Unforgeability. Each credential defines a specific delegation chain. We cannot monitor delegation between adversarial parties. However, we require that whenever the delegation chain shows that an honest player delegated a level $L$ credential to some user, that delegation actually occurred.

In this game, all of the honest parties are controlled by a single oracle that keeps track of all honestly issued credentials. An adversary given access to this oracle should have only negligible probability of outputting a forged credential.

Let $F$ be an efficiently computable bijection and a one-way function. There exists a ppt. algorithms ExtSetup and Extract with five properties:

- ExtSetup and Setup output identically distributed params.

- Under these parameters, pseudonyms are perfectly binding for $s k$.

- Extract always extracts the correct chain of $L$ identities from an honestly generated level $L$ credproof.

- Given an adversarially generated level $L$ credential proof credproof from authority Nym $_{O}$ for the pseudonym Nym, Extract will always produce either the special symbol $\perp$ or $f_{0}, \ldots f_{L}$ such that $N y m_{O}$ is a pseudonym for $F^{-1}\left(f_{0}\right)$ and $N y m$ is a pseudonym for $F^{-1}\left(f_{L}\right)$.

- No adversary can output a valid credential proof from which an unauthorized chain of identities is extracted. More formally we require that for all ppt. $\mathcal{A}$ there exists a negligible $\nu$ such that:

$$
\begin{aligned}
& \operatorname{Pr}\left[\left(\operatorname{params}_{D C}, t d\right) \leftarrow \operatorname{ExtSetup}\left(1^{k}\right) ;\right. \\
& \text { (credproof, Nym, Nym } \left.{ }_{O}, L\right), \leftarrow \mathcal{A}^{\mathcal{O}\left(\text { params }_{D C}, \cdot \cdot \cdot\right)}\left(\text { params }_{D C}, t d\right) \text {; } \\
& \left(f_{0}, \ldots, f_{L}\right) \leftarrow \text { Extract }\left(\text { params }_{D C}, t d \text {, credproof }, \text { Nym }_{1} \text { Nym }_{O}, L\right) \text { : } \\
& \text { CredVerify }\left(\text { params }_{D C}, N y m_{O} \text {, credproof }, N y m, L\right)=\text { accept } \wedge \\
& \left(\exists i \text { such that }\left(f_{0}, i, f_{i-1}, f_{i}\right) \notin \text { ValidCredentialChains } \wedge\right. \\
& \left.\left.f_{i-1} \in \text { HonestUsers }\right)\right] \leq \nu(k),
\end{aligned}
$$

where $\mathcal{O}$ (params $_{D C}$, command, input) describes all possible ways for the adversary $\mathcal{A}$ to interact with the delegatable credentials system: $\mathcal{A}$ can ask the oracle to add new honest users; the oracle generates $s k \leftarrow$ Keygen $\left(\right.$ params $\left._{D C}\right)$, stores it in the list HonestUsers, and returns $F(s k)$ as the handle. $\mathcal{A}$ can ask for new pseudonyms for existing honest users, referenced by $F(s k)$, and he can provide a 
credential and ask an honest user to generate the corresponding proof. Finally, he can run the Issue $\leftrightarrow$ Obtain protocols on credentials of his choice, either between honest users, or with an adversarial issuer or obtainer. In this case, we need to keep track of which credentials are being issued, so that we will be able to identify a forgery. To do this, we use the Extract algorithm to extract the chain of identities behind each credential being issued and store it on the list ValidCredentialChains. For details, see full version $\mathrm{BCC}^{+} 08$.

Remark 3. We let the adversary track honest users' credentials and pseudonyms (but, of course, not their secret keys). Our definition is strictly stronger than one that uses a general oracle that does not reveal the credentials of honest users to the adversary. This approach results in a simpler definition and analysis.

\subsection{Construction of Delegatable Credentials}

We construct delegatable credentials using a randomizable NIZK proof system with randomizable commitments (as described in Section 2) and a message authentication scheme for a vector of messages $\boldsymbol{m}$ (in our basic scheme $|\boldsymbol{m}|=$ 2 )in the common parameters model: AuthSetup $\left(1^{k}\right)$ outputs common parameters $\operatorname{params}_{A}$, AuthKg(params $\left.{ }_{A}\right)$ outputs a secret key sk, Auth $\left(\right.$ params $\left._{A}, s k, \boldsymbol{m}\right)$ outputs an authentication tag auth that authenticates a vector of messages $\boldsymbol{m}$, and VerifyAuth $\left(\right.$ params $_{A}, s k, \boldsymbol{m}$, auth $)$ accepts if auth is a proper authenticator for $\boldsymbol{m}$ under key $s k$. (We will discuss the properties we will require from this authentication scheme after we present our delegatable credentials construction.)

The parameters of the delegatable credentials system combine the parameters params $_{A}$ from the authentication scheme and params $_{P K}$ from the composable and randomizable NIZKPK system and its associated commitment scheme Commit. We assume that all algorithms are aware of these parameters and omit them when appropriate to simplify our notation.

Intuition behind our construction. The keyspace of the authenticator must be a subset of the input space of the commitment scheme. Each user $U$ has a secret key $s k_{U} \leftarrow$ AuthKg $\left(\right.$ params $\left._{A}\right)$, and forms his pseudonyms using Commit: Nym $_{U}=$ Commit $\left(s k_{U}\right.$, open $\left.n_{U}\right)$. $U$ can create arbitrarily many different pseudonyms by choosing new random values open ${ }_{U}$. A user can act as an authority (originator) for credentials by making his pseudonym $N y m_{O}$ publicly available.

The user's secret credential cred is a NIZKPK of a statement about U's specific secret pseudonym $S_{U}=\operatorname{Commit}\left(s k_{U}, 0\right)$ (this specific pseudonym does not in fact hide $s k_{U}$ since it is formed as a deterministic function of $\left.s k_{U}\right)$. To show or delegate the credential, the user randomizes and mauls cred to obtain credproof using the RandProof algorithm described in Section 2. The resulting credproof is a proof about a proper pseudonym, $N y m_{U}=\operatorname{Commit}\left(s k_{U}\right.$, open $)$ for a randomly chosen open.

Suppose a user with secret key $s k_{U}$ has a level $L$ credential from some authority $O$, and let $\left(s k_{O}, s k_{1}, \ldots, s k_{L-1}, s k_{U}\right)$ be the keys such that the owner of $s k_{i}$ delegated the credential to $s k_{i+1}$ (we let $s k_{0}=s k_{O}$ and $s k_{L}=s k_{U}$ ). A 
certification chain is a list of authenticators auth ${ }_{1}, \ldots$, auth $h_{L}$, such that $s k_{i}$ was used to generate authenticator $a u t h_{i+1}$ on message $s k_{i+1}$.

To make sure that pieces of different certification chains cannot be mixed and matched, we add a label $r_{i}$ to each authenticator. The labels have to be unique for each authority and delegation level. Let $H$ be a collision resistant hash function with an appropriate range. For a credential chain rooted at $N y m_{O}$, we set $r_{i}=$ $H\left(\right.$ Nym $\left._{O}, i\right)$. Each $a u t h_{i}$ is then an output of Auth $\left(\right.$ params $\left._{A}, s k_{i-1},\left(s k_{i}, r_{i}\right)\right)$. Let $F$ be an efficiently computable bijection. The user $U$ 's level $L$ private credential cred is a proof of the form

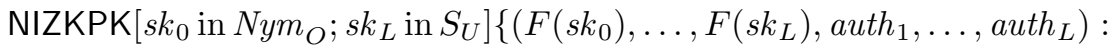

$\operatorname{VerifyAuth}\left(s k_{0},\left(s k_{1}, r_{1}\right)\right.$, auth $\left._{1}\right) \wedge \ldots \wedge \operatorname{VerifyAuth}\left(s k_{L-1},\left(s k_{L}, r_{L}\right)\right.$, auth $\left.\left._{L}\right)\right\}$

Full construction. Let PKSetup, PKProve, PKVerify, and RandProof be a randomizable NIPK system and let AuthSetup, AuthKg, Auth, VerifyAuth be an authentication scheme, and let $H:\{0,1\}^{*} \rightarrow Z_{p}$ be a hash function.

Setup $\left(1^{k}\right)$. Use AuthSetup $\left(1^{k}\right)$ to generate $\operatorname{params}_{A}$ and $\mathrm{PKSetup}\left(1^{k}\right)$ to generate params $_{P K}$; choose the hash function $H$ (as explained above); and output params $_{D C}=\left(\right.$ params $_{A}$, params $\left._{P K}, H\right)$.

Keygen $\left(\right.$ params $\left._{D C}\right)$. Run AuthKg $\left(\right.$ params $\left._{A}\right)$ and output the secret key sk. Nymgen $\left(\right.$ params $\left._{D C}, s k\right)$. Choose random open, compute $N y m=$ Commit params $_{P K}$, sk, open) and output pseudonym Nym and auxiliary information open.

CredProve $\left(\right.$ params $_{D C}$, Nym $_{O}$, cred $, k_{U}, N y m_{U}$ open $\left._{U}, L\right)$. If PKVerify $\left(\right.$ params $_{P K}$, $\left(\right.$ Nym $\left._{O}, \operatorname{Commit}\left(s k_{U}, 0\right)\right)$, cred $)$ rejects, or if $N y m_{U} \neq \operatorname{Commit}\left(\right.$ sk $_{U}$, open $\left._{U}\right)$, abort. Return credproof $\leftarrow$ RandProof $\left(\left(\right.\right.$ Nym $\left._{O}, N y m_{U}\right),\left(0\right.$, open $\left._{U}\right)$, cred $)$.

CredVerify $\left(\right.$ params $_{D C}$, Nym $_{O}$, credproof $\left., N y m_{U}, L\right)$ runs PKVerify.



$\leftrightarrow$ Obtain params $_{D C}$, Nym $_{O}$, sk $_{U}$, Nym $_{U}$, open $\left._{U}, N y m_{I}, L\right)$. Abort if $L=0$ and Nym $_{O} \neq N y m_{I}$. The issuer verifies cred using CredVerify and if it does not verify or if $\operatorname{Nym}_{I} \neq \operatorname{Commit}\left(s_{I}\right.$, open $\left.{ }_{I}\right)$ or $N y m_{U}$ is not a valid pseudonym, the issuer aborts. Else, the issuer and the user both compute $r_{L+1}=$ $H\left(\right.$ Nym $\left._{O}, L+1\right)$. The issuer and the user run a two-party protocol with the following specifications: the public input is $\left(N y m_{I}, N y m_{U}, r_{L+1}\right)$; the issuer's private input is $\left(s k_{I}\right.$, open $\left._{I}\right)$ and the user's private input is $\left(s k_{U}\right.$, open $\left._{U}\right)$. The output of the protocol is as follows: if $\left(s_{I}\right.$, open $\left._{I}\right)$ and $\left(s k_{U}\right.$, open $\left._{U}\right)$ do not appropriately correspond to $N y m_{I}, N y m_{U}$, the protocol aborts; otherwise, the issuer receives no output while the user receives as output the value $\pi$ computed as:

$$
\begin{array}{r}
\pi \leftarrow \operatorname{NIZKPK}\left[s k_{I} \text { in } \operatorname{Nym}_{I} ; s k_{U} \text { in } \operatorname{Commit}\left(s k_{U}, 0\right)\right]\left\{\left(F\left(s k_{I}\right), F\left(s k_{U}\right), \text { auth }\right):\right. \\
\left.\operatorname{VerifyAuth}\left(s k_{I},\left(s k_{U}, r_{L+1}\right), \text { auth }\right)\right\} .
\end{array}
$$

In Section 3.3 we give an efficient instantiation of such a $2 \mathrm{PC}$ protocol for the specific authentication and NIZKPK schemes we use. 
If $L=0$, then the user outputs cred $_{U}=\pi$. Otherwise, the issuer obtains credproof $_{I} \leftarrow$ CredProve $\left(\right.$ params $_{D C}$, Nym $_{O}$, cred sk $_{I}$, Nym $_{I}$, open $\left._{I}, L\right)$ and sends it to the user. Let $S_{U}=\operatorname{Commit}\left(s k_{U}, 0\right)$. Intuitively, credproof $_{I}$ is a proof that the owner of $N y m_{I}$ has a level $L$ credential under public key $N_{y} m_{O}$, while $\pi$ is proof that the owner of $N y m_{I}$ delegated to the owner of $S_{U}$. The user concatenates credproof ${ }_{I}$ and $\pi$ to obtain credproof $_{I} \circ \pi$. To get cred $_{U}$, $U$ needs to project credproof $_{I} \circ \pi$ into a proof about $\left(N_{y m_{O}}, S_{U}\right)$ instead of Nym $_{I}$.

Remark 4. We can attach public attributes to each level of the credential. We compute $r_{\ell}=H\left(s k_{O}, \ell, \operatorname{attr}_{1}, \ldots\right.$, attr $\left.{ }_{\ell}\right)$, where $\operatorname{attr}_{i}$ is the set of attributes added by the $i$ th delegator in the delegation chain. When the user shows or delegates a credential, he must display all the attributes associated with each level.

Message authentication scheme. Just like a signature scheme, an authentication scheme must be complete and unforgeable. For our application we need to strengthen the unforgeability property in two ways. First, we require $F$ Unforgeability BCKL08, which guarantees that for some well-defined bijection $F$, no adversary can output $(F(\boldsymbol{m})$, auth) without first getting an authenticator on $m$. (We write $F(\boldsymbol{m})=F\left(m_{1}, \ldots, m_{n}\right)$ to denote $\left(F\left(m_{1}\right), \ldots, F\left(m_{n}\right)\right)$.) Second we require a new property which we call certification security; the authenticator is unforgeable even if the adversary learns a signature on the challenge secret key. An authentication scheme is $F$-unforgeable and certification secure if for all ppt. adversaries $\mathcal{A}$ there exists negligible $\nu$ such that:

$$
\begin{aligned}
& \operatorname{Pr}\left[\text { params }_{A} \leftarrow \text { AuthSetup }\left(1^{k}\right) ; s k \leftarrow \operatorname{AuthKg}\left(\text { params }_{A}\right) ;\right. \\
& (\boldsymbol{y}, \text { auth }) \leftarrow \mathcal{A}^{\mathcal{O}_{\text {Auth }}\left(\text { params }_{A}, s k, .\right), \mathcal{O}_{\text {Certify }}\left(\operatorname{params}_{A}, .,(s k, ., \ldots)\right)}\left(\operatorname{params}_{A}, F(s k)\right): \\
& \left.\operatorname{VerifyAuth}\left(\operatorname{params}_{A}, s k, F^{-1}(\boldsymbol{y}), \text { auth }\right)=1 \wedge F^{-1}(\boldsymbol{y}) \notin Q_{\text {Auth }}\right] \leq \nu(k) \text {, }
\end{aligned}
$$

where the oracle $\mathcal{O}_{\text {Auth }}\left(\right.$ params $\left._{A}, s k, \boldsymbol{m}\right)$ outputs Auth $\left(\right.$ params $\left._{A}, s k, \boldsymbol{m}\right)$ and stores $\boldsymbol{m}$ on $Q_{\text {Auth }}$, and oracle $\mathcal{O}_{\text {Certify }}\left(\right.$ params $\left._{A}, s k^{*},\left(s k, m_{2}, \ldots, m_{n}\right)\right)$ outputs the signature Auth $\left(\right.$ params $\left._{A}, s k^{*},\left(s k, m_{2}, \ldots, m_{n}\right)\right)$.

Theorem 1. Let AuthSetup, AuthKg, Auth, VerifyAuth be an F-unforgeable certification-secure authentication scheme, $H$ be a collision resistant hash function, and PKSetup, PKProve, PKVerify be a randomizable, partially extractable, composable zero-knowledge non-interactive proof of knowledge system. Then the above construction constitutes a secure anonymous delegatable credential scheme. See full version $\left[B C C^{+} 08\right]$ for proof.

We will construct our authentication scheme based the BB-CDH and BB-HSDH assumptions (defined in Section 3.3). Groth-Sahai proofs require either the SXDH assumption or the Decision Linear Assumption GS08. Our two party protocol requires a homomorphic encryption scheme. 


\subsection{Building Block Instantiations}

Bilinear Maps and Assumptions. We use standard notation for groups with a computable bilinear map $e: G_{1} \times G_{2} \rightarrow G_{T}$. See, e.g., BLS04, GPS06. The security of our scheme is based on strengthened versions of the SDH [BB04] and $\mathrm{CDH}$ assumptions. BB-CDH is implied by SDH; Boy08 describes how to extend Generalized Diffie Hellman BBG05] to cover these two assumptions and prove their generic group security.

Definition 3 (BB-HSDH). Let $x, c_{1} \ldots c_{q} \leftarrow Z_{p}$. On input $g, g^{x}, u \in G_{1}$, $h, h^{x} \in G_{2}$ and the tuple $\left\{g^{1 /\left(x+c_{\ell}\right)}, c_{\ell}\right\}_{\ell=1 \ldots q}$, it is computationally infeasible to output a new tuple $\left(g^{1 /(x+c)}, h^{c}, u^{c}\right)$.

Definition 4 (BB-CDH). Let $x, y, c_{1} \ldots c_{q} \leftarrow Z_{p}$. On input $g, g^{x}, g^{y} \in G_{1}$, $h, h^{x} \in G_{2}$ and the tuple $\left\{g^{1 /\left(x+c_{\ell}\right)}, c_{\ell}\right\}_{\ell=1 \ldots q}$, it is computationally infeasible to output $g^{x y}$.

F-Unforgeable Certification Secure Message Authentication Scheme. Our authentication scheme is based on the Boneh-Boyen weak signature scheme [BB04, where $\operatorname{Sign}_{s k}(m)=g^{1 /(s k+m)}$. Belenkiy et al. showed that the Boneh-Boyen signature scheme is $F$-unforgeable for the bijection $F(m)=\left(g^{m}, u^{m}\right.$ ) (under a very strong assumption), and that the Groth-Sahai proof system can be used to prove knowledge of such a signature. Boneh-Boyen signatures are not certification secure because $\operatorname{Sign}_{s k}(m)=\operatorname{Sign}_{m}(s k)$. We show how to achieve certification security; we also authenticate a vector of messages and weaken the underlying security assumption. The construction is as follows: Auth $\left(s k, m_{1} \| m_{2}\right)$ chooses random keys $K^{*}, K_{1}, K_{2}$ and returns $\left(\operatorname{Sign}_{s k}\left(K^{*}\right), \operatorname{Sign}_{K^{*}}\left(K_{1}\right), \operatorname{Sign}_{K^{*}}\left(K_{2}\right)\right.$, $\left.\operatorname{Sign}_{K_{1}}\left(m_{1}\right), \operatorname{Sign}_{K_{2}}\left(m_{2}\right), F\left(K^{*}\right), F\left(K_{1}\right), F\left(K_{2}\right)\right)$. At a high level, this construction eliminates any symmetries between $\operatorname{Auth}_{s k}(m)$ and $\operatorname{Auth}_{m}(s k)$. See full version $\left[\mathrm{BCC}^{+} 08\right.$ for details.

Theorem 2. The message authentication scheme above is $F$-unforgeable and certification secure for $F\left(m_{i}\right)=\left(h^{m_{i}}, u^{m_{i}}\right)$ under the BB-HSDH and BB-CDH assumptions. See full version $\mathrm{BCC}^{+} 08$ for proof. The signature scheme obtained by setting $p k=h^{s k}$ may be of independent interest.

Commitment scheme. A commitment to $x \in Z_{p}$ consists of two GS commitments GSCommit $\left.\left(h^{x}, o_{1}\right), \operatorname{GSCommit}\left(u^{x}, o_{2}\right)\right)$ and a $\operatorname{NIPK}_{G S}$ proof that these are commitments to the same value $x$. This allows us to extract $F(x)=\left(h^{x}, u^{x}\right)$.

Proof of knowledge of an authenticator. We need a NIZKPK of an authenticator for messages $\boldsymbol{m}=\left(m_{1}, m_{2}\right)$, where the first value is hidden in commitment $C_{m_{1}}$ and the second value $m_{2}$ is publicly known. In our notation, this is:

$$
\begin{aligned}
& \text { NIZKPK }\left[s k \text { in } C_{s k} ; m_{1} \text { in } C_{m_{1}}\right]\left\{\left(F(s k), F\left(m_{1}\right), \text { auth }\right):\right. \\
&\text { VerifyAuth } \left.\left(\text { params }_{A}, s k,\left(m_{1}, m_{2}\right), \text { auth }\right)=1\right\} .
\end{aligned}
$$

Since Boneh-Boyen signatures are verified using pairing product equations, we can use Groth-Sahai proofs, see full version $\left[\mathrm{BCC}^{+} 08\right.$ for details. 
Creating a NIZKPK of an authenticator. The issuer chooses $K^{*}, K_{1}, K_{2}$ and can generate most of the proof. Then, the issuer and user need to jointly compute a NIZKPK of a Boneh-Boyen signature on the user's secret key. We outline the protocol, see full version $\left[\mathrm{BCC}^{+} 08\right.$ for details.

Let Keygen, Enc, Dec be an additively homomorphic semantically secure encryption scheme, let " $\oplus$ " denote the homomorphic operation on ciphertexts; for $e$ a ciphertext and $r$ an integer, $e \otimes r$ denotes "adding" $e$ to itself $r$ times. The user with input $m_{1}$, and the issuer with input $K_{1}$ run the following protocol to

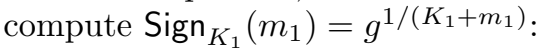

1. The issuer generates $\left(s k_{h o m}, p k_{h o m}\right) \leftarrow \operatorname{Keygen}\left(1^{k}\right)$ in such a way that the message space is of size at least $2^{k} p^{2}$. He then computes $e_{1}=\operatorname{Enc}\left(p k_{h o m}, K_{1}\right)$ and sends $e_{1}, p k_{h o m}$ to the user and engages with her in an interactive zeroknowledge proof that $e_{1}$ encrypts to a message in $[0, p]$.

2. The user chooses $r_{1} \leftarrow Z_{p}$ and $r_{2} \leftarrow\left\{0, \ldots, 2^{k} p\right\}$, then computes $e_{2}=$ $\left(\left(e_{1} \oplus \operatorname{Enc}\left(p k_{h o m}, m_{1}\right)\right) \otimes r_{1}\right) \oplus \operatorname{Enc}\left(p k_{h o m}, r_{2} p\right)$ and sends $e_{2}$ to the user.

3 . The issuer and the user perform an interactive zero-knowledge proof in which the user shows that $e_{2}$ has been computed correctly using the message in $C_{m_{1}}$, and that $r_{1}, r_{2}$ are in the appropriate ranges.

4. The issuer decrypts $x=\operatorname{Dec}\left(s k_{h o m}, e_{2}\right)$, sends the user $\sigma^{*}=g^{1 / x}$.

5. The user computes $\sigma=\sigma^{* r_{1}}$ and verifies that it is a correct weak BB signature on $m_{1}$. The issuer obtains no information about $m_{1}$.

Theorem 3. The above is a secure two-party computation for computing BonehBoyen signatures. (See full version $\left[\mathrm{BCC}^{+} 08\right]$ for proof.)

Acknowledgements. Jan Camenisch was supported in part by the European Commission through the ICT and IST programmes under contract ICT-216483 PRIMELIFE. Markulf Kohlweiss was supported in part by the Concerted Research Action (GOA) Ambiorics 2005/11 of the Flemish Government, by the IAP Programme P6/26 BCRYPT of the Belgian State (Belgian Science Policy), and in part by the European Commission through the ICT and IST programmes under the following contracts: ICT-216483 PRIMELIFE and ICT-216676 ECRYPT II. Hovav Shacham was supported by an AFOSR MURI grant and, while at the Weizmann Institute of Science, by a Koshland Scholars Program postdoctoral fellowship. Mira Belenkiy, Melissa Chase and Anna Lysyanskaya acknowledge the support of NSF grants 0831293, 0627553 and 0347661. The information in this document reflects only the authors' views.

\section{References}

[Bar01] Barak, B.: Delegatable signatures. Technical report, Weizmann Institute of Science (2001)

[BB04] Boneh, D., Boyen, X.: Short signatures without random oracles. In: Cachin, C., Camenisch, J.L. (eds.) EUROCRYPT 2004. LNCS, vol. 3027, pp. 56-73. Springer, Heidelberg (2004) 
[BBG05] Boneh, D., Boyen, X., Goh, E.: Hierarchical identity based encryption with constant size ciphertext. In: Cramer, R. (ed.) EUROCRYPT 2005. LNCS, vol. 3494, pp. 440-456. Springer, Heidelberg (2005)

$\left[\mathrm{BCC}^{+} 08\right]$ Belenkiy, M., Camenisch, J., Chase, M., Kohlweiss, M., Lysyanskaya, A., Shacham, H.: Randomizable proofs and delegatable anonymous credentials. Cryptology ePrint Archive, Report 2008/428 (2008), http://eprint.iacr.org/2008/428

[BCKL08] Belenkiy, M., Chase, M., Kohlweiss, M., Lysyanskaya, A.: P-signatures and noninteractive anonymous credentials. In: Canetti, R. (ed.) TCC 2008. LNCS, vol. 4948, pp. 356-374. Springer, Heidelberg (2008)

[BDI ${ }^{+99}$ Burmester, M., Desmedt, Y., Itoh, T., Sakurai, K., Shizuya, H.: Divertible and subliminal-free zero-knowledge proofs for languages. Journal of Cryptology 12(3), 197-223 (1999)

[BDMP91] Blum, M., De Santis, A., Micali, S., Persiano, G.: Non-interactive zeroknowledge. SIAM Journal of Computing 20(6), 1084-1118 (1991)

[BFM88] Blum, M., Feldman, P., Micali, S.: Non-interactive zero-knowledge and its applications. In: STOC 1988 (1988)

[BLS04] Boneh, D., Lynn, B., Shacham, H.: Short signatures from the Weil pairing. J. Cryptology 17(4), 297-319 (2004)

[Boy08] Boyen, X.: The uber-assumption family. In: Galbraith, S.D., Paterson, K.G. (eds.) Pairing 2008. LNCS, vol. 5209, pp. 39-56. Springer, Heidelberg (2008)

[Bra99] Brands, S.: Rethinking Public Key Infrastructure and Digital CertificatesBuilding in Privacy. PhD thesis, Eindhoven Inst. of Tech. The Netherlands (1999)

[Cha85] Chaum, D.: Security without identification: Transaction systems to make big brother obsolete. Communications of the ACM 28(10), 1030-1044 (1985)

$\left[\mathrm{CHK}^{+} 06\right]$ Camenisch, J., Hohenberger, S., Kohlweiss, M., Lysyanskaya, A., Meyerovich, M.: How to win the clonewars: efficient periodic n-times anonymous authentication. In: CCS 2006 (2006)

[CL01] Camenisch, J., Lysyanskaya, A.: Efficient non-transferable anonymous multi-show credential system with optional anonymity revocation. In: Eurocrypt 2001 (2001)

[CL02a] Camenisch, J., Lysyanskaya, A.: Dynamic accumulators and application to efficient revocation of anonymous credentials. In: Yung, M. (ed.) CRYPTO 2002. LNCS, vol. 2442, p. 61. Springer, Heidelberg (2002)

[CL02b] Camenisch, J., Lysyanskaya, A.: A signature scheme with efficient protocols. In: Cimato, S., Galdi, C., Persiano, G. (eds.) SCN 2002. LNCS, vol. 2576, pp. 268-289. Springer, Heidelberg (2003)

[CL06] Chase, M., Lysyanskaya, A.: On signatures of knowledge. In: Dwork, C. (ed.) CRYPTO 2006. LNCS, vol. 4117, pp. 78-96. Springer, Heidelberg (2006)

[CS97] Camenisch, J., Stadler, M.: Efficient group signature schemes for large groups. In: Kaliski Jr., B.S. (ed.) CRYPTO 1997. LNCS, vol. 1294, pp. 410-424. Springer, Heidelberg (1997)

[Dam02] Damgård, I.: On $\sigma$-protocols (2002), http://www.daimi.au.dk/ ivan/Sigma.ps

[DSY90] De Santis, A., Yung, M.: Cryptographic applications of the non-interactive metaproof and many-prover systems. In: Menezes, A., Vanstone, S.A. (eds.) CRYPTO 1990. LNCS, vol. 537, pp. 366-377. Springer, Heidelberg (1991)

[FLS99] Feige, U., Lapidot, D., Shamir, A.: Multiple noninteractive zero knowledge proofs under general assumptions. SIAM Journal on Computing 29(1), 1-28 (1999) 
[GMR89] Goldwasser, S., Micali, S., Rackoff, C.: The knowledge complexity of interactive proof systems. SIAM J. Comput. 18(1), 186-208 (1989)

[Gol00] Goldreich, O.: Foundations of Cryptography: Basic Tools. Cambridge University Press, New York (2000)

[GOS06] Groth, J., Ostrovsky, R., Sahai, A.: Perfect non-interactive zero knowledge for np. In: Vaudenay, S. (ed.) EUROCRYPT 2006. LNCS, vol. 4004, pp. 339-358. Springer, Heidelberg (2006)

[GPS06] Galbraith, S., Paterson, K., Smart, N.: Pairings for cryptographers. Cryptology ePrint Archive, Report 2006/165 (2006), http://eprint.iacr.org/

[GS02] Gentry, C., Silverberg, A.: Hierarchical ID-based cryptography. In: Zheng, Y. (ed.) ASIACRYPT 2002. LNCS, vol. 2501, pp. 548-566. Springer, Heidelberg (2002)

[GS08] Groth, J., Sahai, A.: Efficient non-interactive proof systems for bilinear groups. In: Smart, N.P. (ed.) EUROCRYPT 2008. LNCS, vol. 4965, pp. 415-432. Springer, Heidelberg (2008)

[KP98] Kilian, J., Petrank, E.: An efficient non-interactive zero-knowledge proof system for np with general assumptions. J. of Cryptology (1998)

[LRSW99] Lysyanskaya, A., Rivest, R.L., Sahai, A., Wolf, S.: Pseudonym systems. In: Heys, H.M., Adams, C.M. (eds.) SAC 1999. LNCS, vol. 1758, p. 184. Springer, Heidelberg (2000)

[SCP00] De Santis, A., Di Crescenzo, G., Persiano, G.: Necessary and sufficient assumptions for non-interactive zero-knowledge proofs of knowledge for all NP relations. In: Welzl, E., Montanari, U., Rolim, J.D.P. (eds.) ICALP 2000. LNCS, vol. 1853, p. 451. Springer, Heidelberg (2000) 